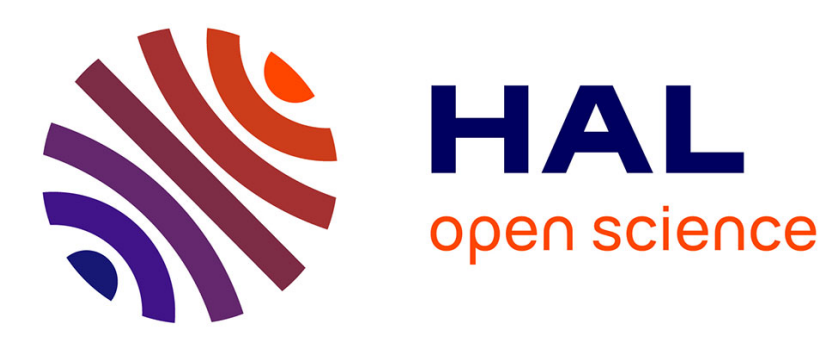

\title{
Clonal analysis by tunable CRISPR-mediated excision
} Anna F Gilles, Johannes B Schinko, Magdalena I Schacht, Camille Enjolras, Michalis Averof

\section{To cite this version:}

Anna F Gilles, Johannes B Schinko, Magdalena I Schacht, Camille Enjolras, Michalis Averof. Clonal analysis by tunable CRISPR-mediated excision. Development (Cambridge, England), 2019, 10.1242/dev.170969 . hal-02327429

\section{HAL Id: hal-02327429 \\ https://hal.science/hal-02327429}

Submitted on 22 Oct 2019

HAL is a multi-disciplinary open access archive for the deposit and dissemination of scientific research documents, whether they are published or not. The documents may come from teaching and research institutions in France or abroad, or from public or private research centers.
L'archive ouverte pluridisciplinaire HAL, est destinée au dépôt et à la diffusion de documents scientifiques de niveau recherche, publiés ou non, émanant des établissements d'enseignement et de recherche français ou étrangers, des laboratoires publics ou privés. 


\title{
Clonal analysis by tunable CRISPR-mediated excision
}

\author{
Anna F. Gilles ${ }^{1,2,3, *}$, Johannes B. Schinko ${ }^{1,3,4, *}$, Magdalena I. Schacht ${ }^{1,5}$, Camille Enjolras ${ }^{1,4}$ and \\ Michalis Averof ${ }^{1,4, *}$
}

\begin{abstract}
Clonal marking techniques based on the Cre/lox and Flp/FRT systems are widely used in multicellular model organisms to mark individual cells and their progeny, in order to study their morphology, growth properties and developmental fates. The same tools can be adapted to introduce specific genetic changes in a subset of cells within the body, i.e. to perform mosaic genetic analysis. Marking and manipulating distinct cell clones requires control over the frequency of clone induction, which is sometimes difficult to achieve. Here, we present Valcyrie, a new method that replaces the conventional Cre or Flp recombinase-mediated excision of a marker cassette by CRISPR-mediated excision. A major advantage of this approach is that CRISPR efficiency can be tuned in a predictable fashion by manipulating the degree of sequence complementarity between the CRISPR guide RNA and its targets. We establish the method in the beetle Tribolium castaneum. We demonstrate that clone marking frequency can be tuned to generate embryos that carry single marked clones. The Valcyrie approach can be applied to a wide range of experimental settings, for example to modulate clone frequency with existing tools in established model organisms and to introduce clonal analysis in emerging experimental models.
\end{abstract}

KEY WORDS: CRISPR, Tribolium, Clonal analysis, Genetic mosaics, Insect

\section{INTRODUCTION}

Clonal analysis originates from efforts to track the fate of individual cells and their progeny during development (reviewed by Kretzschmar and Watt, 2012). Early studies used cell transplantation from genetically distinct donors or labelling using vital dyes to track the fate of marked cells. Spontaneous somatic mutations that produce a visible cellular phenotype, such as the loss of pigmentation, were also used to observe the territories occupied by cell clones in mosaic animals and plants. In principle, any heritable somatic change that is associated with a visible marker can be used for this purpose, and different types of genetic events have been exploited for this, including chromosome loss, transposon excision, genetic rearrangement and mitotic recombination (Garcia-Bellido et al., 1973; Janning, 1978; Nicolas et al., 1996; Stern, 1936; Vincent et al., 1995).

\footnotetext{
${ }^{1}$ Institut de Génomique Fonctionnelle de Lyon (IGFL), École Normale Supérieure de Lyon, 32 avenue Tony Garnier, 69007 Lyon, France. ${ }^{2}$ BMIC graduate programme, Université Claude Bernard/Lyon 1, France. ${ }^{3}$ TriGenes gUG, Biberach University of Applied Sciences, Hubertus-Liebrecht-Str. 35, 88400 Biberach/Riss, Germany. ${ }^{4}$ Centre National de la Recherche Scientifique (CNRS), France. ${ }^{5}$ Department of Evolutionary Developmental Genetics, Universität Göttingen, Justus-von-LiebigWeg 11, 37077 Göttingen, Germany.

*Authors for correspondence (anna.gilles@trigenes.com; johannes.schinko@ trigenes.com; michalis.averof@ens-lyon.fr)

(D) A.F.G., 0000-0001-5491-3516; J.B.S., 0000-0002-2985-8006; M.A., 0000-00026803-7251
}

Received 21 August 2018; Accepted 26 November 2018
Clonal analysis received a great boost with the introduction of recombinases and fluorescent proteins in the 1990s: recombinases made the task of inducing genetic rearrangements more efficient and easier to engineer (Golic and Lindquist, 1989; Orban et al., 1992), and fluorescent proteins provided versatile markers for visualising the cell clones. The most common method for marking cell clones, used until today, consists of expressing a recombinase (bacteriophage-derived Cre or yeast-derived Flp) in the cells of interest, in which it induces recombination among pairs of the respective target site (lox or FRT). These target pairs can be placed either in tandem, to excise the intervening DNA fragment containing a marker (flip-out), or on homologous chromosomes, to exchange chromosome arms carrying markers and/or mutations. Many elaborations of those two configurations have been used, varying in the number and complexity of genetic rearrangements (reviewed by Griffin et al., 2014; Richier and Salecker, 2014).

Today, clonal marking techniques are widely applied to visualise the morphology and behaviour of single cells within complex tissues, and to investigate lineage relationships, stem cell dynamics and tissue growth (e.g. Blanpain and Simons, 2013; Chen et al., 2016; Kuchen et al., 2012; Livet et al., 2007; Morante and Desplan, 2008; Snippert et al., 2010). The same tools have also been adapted to create genetic mosaics in which specific genetic changes are introduced in some cells of the body. Such genetic mosaics have been invaluable for discovering the cells in which a gene function is needed, for distinguishing the cell autonomous and non-autonomous functions of a gene, and for studying the effects of mutations that would be lethal in the entire organism (e.g. Hotta and Benzer, 1972; Lee and Luo, 1999; Rajewsky et al., 1996). By allowing us to juxtapose cells of different genotypes in a tissue, mosaics have led to the discovery of genes that underpin key cellcell interactions during development and tissue homeostasis (e.g. Brook et al., 1996; Meyer et al., 2014).

Clonal analysis techniques require good control over the frequency of clone induction. If the frequency is too low, a very large number of individuals must be screened in order to find suitable cell clones to analyse and experiments become very laborious. On the other hand, if the frequency is too high, independent cell clones frequently merge with each other and information on the clonal relationships of cells is lost. In established model organisms, such as Drosophila and mice, the research community has developed and fine-tuned these techniques over decades, generating tools with clone induction frequencies that are well suited for the purpose. When establishing these tools in new experimental models, however, adjusting these frequencies can be laborious and frustrating. Here, we present the difficulties that we faced while establishing clonal analysis tools in the beetle Tribolium castaneum. We show that the Cre/lox system has such high activity in Tribolium that a large number of marked cell clones are generated even by very low (undetectable) levels of Cre expression, driven by the leaky activity of a heat-shock promoter.

To overcome this problem and to optimise the frequency of induction of marked clones, we developed an alternative method for 
generating marked clones, based on the excision of a marker cassette by the CRISPR gene editing system (Cong et al., 2013; Gilles and Averof, 2014; Jinek et al., 2012; Mali et al., 2013), rather than by Cre- or Flp-mediated recombination (Fig. 1). This approach, which we name Valcyrie (for Versatile and adjustable labelling of clones by random CRISPR-induced excision), offers the major advantage that the efficiency of clone induction can be adjusted by manipulating the degree of sequence complementarity between the CRISPR guide RNA (gRNA) and its targets. We demonstrate the effectiveness of this approach in Tribolium. In established model organisms, this tunable method can be readily applied on existing flip-out lines that have been established with constructs carrying lox, $F R T$ or $a t t P / B$ recombinase sites.

\section{RESULTS}

\section{Activity and leakiness of the Cre/lox system in Tribolium}

To establish the Cre/lox system for clonal analysis in T. castaneum, we generated stable transgenic lines bearing conventional Cre and lox flip-out constructs. The Cre-expressing line (Tc-hsp 68:Cre) carries the coding sequence of the Cre recombinase that is driven by the heat-inducible promoter of the Tc-hsp68 gene (hereafter hsp68) (Schinko et al., 2012). The flip-out construct, pUb:lox-mYFP-lox$H 2 B m C h e r r y$, carries a flip-out cassette that consists of the coding sequence of membrane-localised Yellow Fluorescent Protein (mYFP) flanked by loxN sites (Livet et al., 2007), followed by a fusion of coding sequences for the fluorescent protein mCherry (Shaner et al., 2004) and histone H2B of Drosophila (H2B-mCherry); the flip-out construct is transcribed by the ubiquitous $p U b$ promoter (see Materials and Methods). In the absence of a Cre transgene, embryos that carry the flip-out construct show ubiquitous expression of mYFP in late blastoderm and early germband stages (Fig. 2A).

By crossing these transgenic lines, we obtained embryos that carry both the heat-inducible Cre and the flip-out construct. In these embryos, a large number of cells lacked mYFP expression and instead expressed H2B-mCherry, indicating that the lox-mYFP-lox cassette was efficiently excised in the presence of Cre (Fig. 2B,C). We expected the excision to depend on the induction of Cre by a heat shock, but in fact we observed that embryos carry a large number of H2B-mCherry-expressing cells both with and without heat shock. These results suggest that sufficient Cre activity is present even in the absence of a heat shock, because of leaky activity of the hsp 68 promoter. In the absence of heat shock, Cre mRNA was not detectable by in situ hybridisation, which suggests that very low levels of Cre are sufficient to mobilise the lox-mYFP-lox cassette. This Cre activity was present when Cre was provided either maternally or zygotically. Leaky activity of Cre has also been reported in similar experiments carried out in Drosophila (Frickenhaus et al., 2015).

\section{Design of Valcyrie}

In standard Cre/lox flip-out constructs, the expression of Cre drives recombination of paired lox sites, excising a marker gene and bringing a sequence encoding a second marker or gene of interest under the control of a ubiquitous promoter. In the Valcyrie approach, the same flip-out construct can be used, but excision is mediated by a pair of double-strand breaks targeted to the lox sites by CRISPR (Fig. 1). The induction of double-strand breaks is dependent on the expression of the Cas9 protein and a specific gRNA, which together constitute the CRISPR endonuclease (Hwang et al., 2013; Jinek et al., 2012). Once the CRISPR endonuclease has cleaved the two lox sites, the DNA repair mechanisms of the cell join the broken DNA ends; the cleaved sites may be repaired precisely, carry small mutations, or lack the entire fragment between the two targeted sites.

This design provides the opportunity to modulate the production of marked clones in different ways. First, the promoters used to express the Cas9 and the gRNA can influence when, where and at what frequency the marked clones are induced. Here, to test this approach, we used the $h s p 68$ promoter to drive strong expression of Cas9 upon heat shock, and two constitutive RNA polymerase III promoters (Tribolium U6a or U6b; Gilles et al., 2015) to drive the expression of gRNAs.

Second, the level of CRISPR activity can be tuned down by manipulating the degree of sequence complementarity between the

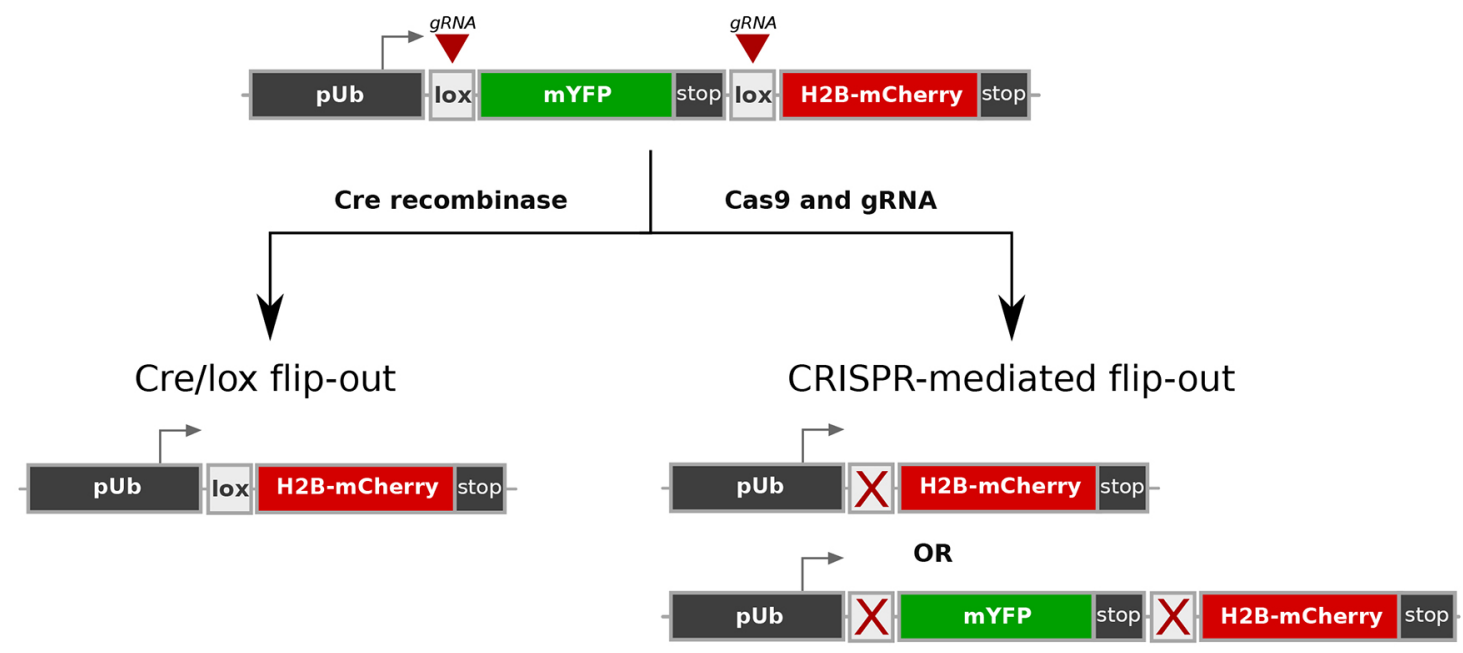

Fig. 1. Recombinase- and CRISPR-mediated approaches for clonal analysis. In conventional recombinase-mediated approaches, the action of a recombinase drives a recombination event that stably marks the cell and its clonal progeny. Here, the Cre recombinase acts on a pair of lox sites, causing the excision of mYFP and bringing H2B-mCherry under the control of the $p U b$ promoter. In the CRISPR-mediated approach, the same excision is caused by double-strand DNA breaks that are generated by the action of Cas9 and gRNAs targeting the lox sites. The CRISPR-mediated approach can also generate point mutations, which prevent further CRISPR activity on the lox sites. The CRISPR-mediated approach allows us to control the timing and frequency of excision by manipulating the expression of Cas 9 and the degree of sequence complementarity between the CRISPR gRNAs and their targets. Mutated lox sites are indicated by a red cross; transcriptional termination and polyadenylation signals are indicated as 'stop'. 

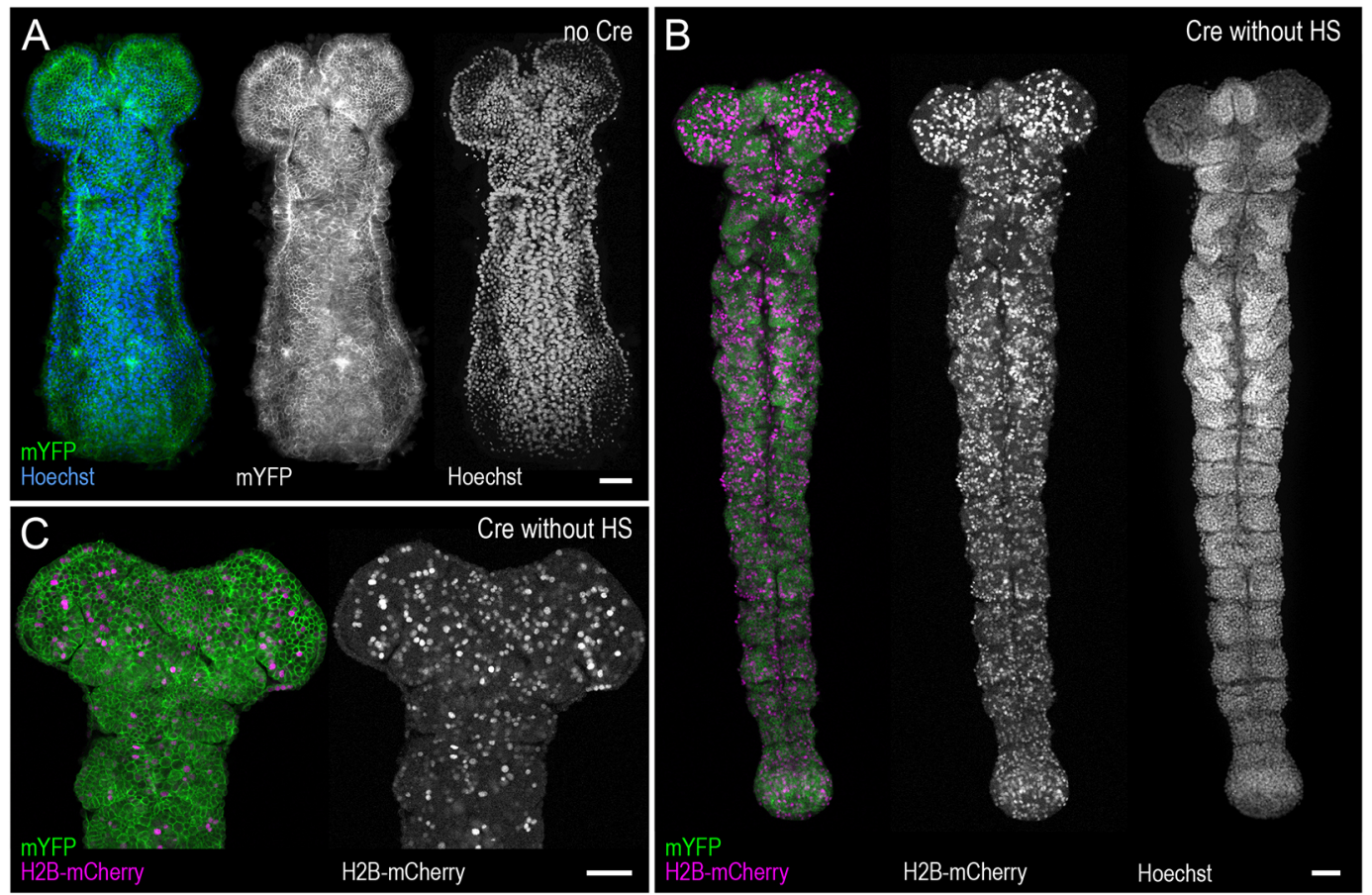

Fig. 2. Leakiness of the Cre/lox system in Tribolium. (A) Uniform expression of mYFP (green) in Tribolium embryo carrying the $p U b:$ lox-mYFP-lox$\mathrm{H} 2 \mathrm{Bm}$ Cherry flip-out construct, in the absence of a Cre transgene. The embryo was also stained with Hoechst 34580 to visualise all nuclei (blue). The mYFP and Hoechst channels are shown separately at the right of the panel. (B-C) mYFP (green) and H2B-mCherry (magenta) expression in Tribolium embryos carrying the same flip-out construct in the presence of the Tc-hsp68:Cre transgene. $\mathrm{H} 2 \mathrm{~B}$-mCherry-marked nuclei are visible even in the absence of a heat shock to induce the expression of Cre (animals were kept at $32^{\circ} \mathrm{C}$ ). The $\mathrm{H} 2 \mathrm{~B}-\mathrm{mCherry}$ channel and Hoechst 34580 staining (in $\mathrm{B}$ ) are shown separately at the right of each panel. Panels show the entire germband (B) and head region (C) of separate embryos. HS, heat shock. Scale bars: $50 \mu \mathrm{m}$.

gRNA and its targets on the flip-out construct. The CRISPR system is known to tolerate mismatches, especially towards the $5^{\prime}$ end of the gRNA (Hsu et al., 2013; Jiang et al., 2013; Fu et al., 2013). With this in mind, we designed and tested two sets of gRNAs that were expected to target the lox sites of our flip-out construct with different efficiencies. First, we designed a pair of gRNAs to target each lox site as efficiently as possible (given the sequence constraints of the flip-out construct, see Materials and Methods): $g R N A$ Lox.R fully matches one of the lox sites, and gRNA Lox.L matches the second lox site with a single nucleotide mismatch at the $5^{\prime}$ end of the gRNA (Fig. 3B). Second, we designed a single gRNA that is expected to target both lox sites with a lower efficiency: $g R N A$ Lox. Uni carries two mismatches with each target, located within the first six nucleotides of the gRNA (Fig. 3B).

Besides generating pairs of double-strand breaks, leading to the excision of the lox-mYFP-lox cassette, we expect that, in some cells, CRISPR activity will generate point mutations (indels) in individual lox sites. The majority of these mutations will produce modified lox sequences that can no longer be targeted by CRISPR (Fig. 1). As these mutations accumulate, they should lead to a decline and eventually an arrest of clone-marking excisions, producing mosaic animals in which only a small number of cells have been marked, and the majority of cells remain unlabelled (carrying mutated lox sites). This would be beneficial for clonal analysis.

To facilitate the genetic crosses bringing the Cas9, gRNA and flip-out elements together, we cloned the Cas9 transgene and each set of gRNAs in the same vector (see Fig. 3A) and generated two independent transgenic lines for each construct, termed Valcyrie. $L R$ \#22 and \#39 (expressing the Lox.L and Lox.R gRNAs) and Valcyrie.Uni \#6 and \#11 (expressing the Lox.Uni gRNA). The lines carry transgene insertions that segregate as single Mendelian loci.
Valcyrie.LR \#22, Valcyrie.Uni \#6 and Valcyrie.Uni \#11 are kept as viable homozygous lines. The insertion present in Valcyrie.LR \#39 is homozygous lethal and it is therefore kept in heterozygous condition.

\section{Generating marked clones at adjustable frequencies via CRISPR-mediated excision}

To test whether CRISPR-mediated excision is effective in generating marked clones, we crossed beetles from each of the Valcyrie.LR and Valcyrie.Uni lines to beetles carrying the flip-out construct $p U b$ :lox-mYFP-lox-H2BmCherry (the same flip-out line that we previously tested for Cre-mediated excision). We collected mixed embryonic stages, from early to late germband stages, and subjected them to a heat shock $\left(46^{\circ} \mathrm{C}\right.$ for $10 \mathrm{~min}$, see Schinko et al., 2012) to induce the expression of Cas9. We let these embryos develop for an additional $12 \mathrm{~h}$ at $32^{\circ} \mathrm{C}$ before fixation and screened them for H2B-mCherry expression. As a control, we screened embryos from identical crosses that were not heat shocked, but were collected and fixed as described above. Ten to 25 embryos from each cross were screened by confocal microscopy.

We detected H2B-mCherry-positive cells in embryos from all four crosses with variable frequency after heat shock (Figs 4 and 5, Table S1). A higher proportion of embryos with visible clones was recorded with the Valcyrie.LR \#22 and \#39 lines ( $>80 \%$ of embryos carrying the construct) than with the Valcyrie.Uni \#6 and \#11 lines (20-70\% of embryos carrying the construct). This result is in line with our expectation that nucleotide mismatches of increasing severity in the gRNAs will generate flip-out clones at lower frequencies. We did not find any H2B-mCherry-positive cells in the controls for the Valcyrie.LR \#22, Valcyrie.Uni \#6 and Valcyrie.Uni \#11 crosses, but we detected some marked cells in the control for Valcyrie.LR \#39, 
A
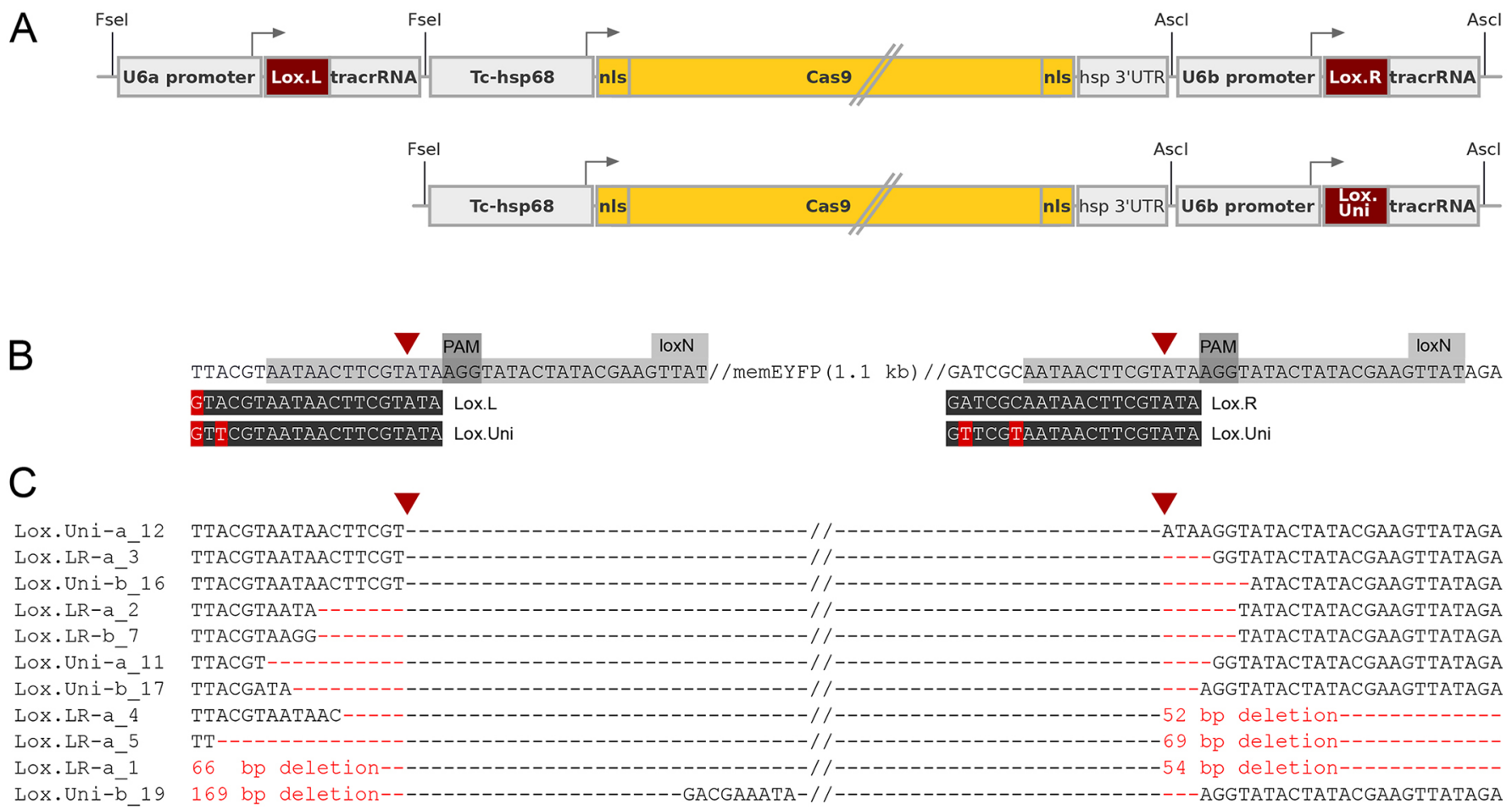

Fig. 3. CRISPR-mediated targeting of lox sites. (A) Schematic of the Valcyrie.LR and Valcyrie.Uni constructs (top and bottom, respectively). (B) Sequence of the loxN sites present in the flip-out construct $p U b$ :Iox-mYFP-lox-H2BmCherry and the targeting sequences of the Lox.L, Lox.R and Lox.Uni gRNAs.

CRISPR targeting requires the presence of a protospacer adjacent motif (PAM) (NGG) in the target sequence. The single PAM found in loxN sites constrains the design of the gRNAs, which must be placed at the edge on the lox site, encompassing 14 nucleotides of the lox site and six nucleotides of the sequence flanking each lox site. The mismatches between the target sites and the gRNAs are marked in red. The CRISPR cleavage site is indicated by an arrowhead. (C) Sequences that resulted from independent excision events (aligned to the target sequences). The excision events are usually imprecise, as expected for repair by non-homologous end joining. In four cases the deletions extended upstream or downstream of the targeted lox sites.

indicating leaky expression of Cas9 in this line, in the absence of a heat shock (data not shown). These results indicate that most Valcyrie lines give us control over the induction of marked clones.

In addition to scoring the presence or absence of H2B-mCherryexpressing cells, we also counted how many distinct clusters of H2B-mCherry-positive cells could be found in each embryo (Fig. 4D, Table S1). We used the number of these clusters as a proxy of the number of independent cell clones marked in each embryo. As expected, embryos from the Valcyrie.LR lines have more clusters than embryos from the Valcyrie.Uni lines (Fig. 4D), which we attribute to the different efficiency of the two sets of gRNAs. However, there is also a considerable difference between the two lines carrying each construct, which is likely due to different levels of Cas9 and gRNAs produced from each transgene insertion site.

These results show that introducing mismatches between the gRNAs and the lox target sites is an effective strategy for reducing the frequency of marked clones in a predictable fashion.

Based on previous work (Schinko et al., 2012), we expected that the temperature and/or the duration of the heat shock could influence the expression level of Cas9, and therefore provide an additional means for tuning the frequency of clone labelling to desired levels. To investigate this possibility, we repeated the experiment described above with a milder heat shock, at $44^{\circ} \mathrm{C}$ instead of $46^{\circ} \mathrm{C}$. As anticipated, we found that the number of marked cell clusters was greatly reduced with all four Valcyrie lines (Fig. 4D, Table S1B).

Further, we suspected that the number of marked clones might also depend on the incubation temperature after the heat shock. To probe this, we collected embryos from the Valcyrie.LR $\# 22$ line at $32^{\circ} \mathrm{C}$ and subjected them to a heat shock for $10 \mathrm{~min}$ at $46^{\circ} \mathrm{C}$. Then we let one batch of these embryos develop at $32^{\circ} \mathrm{C}$ for $20 \mathrm{~h}$, and another at $25^{\circ} \mathrm{C}$ for $40 \mathrm{~h}$. Tribolium development is approximately twice as fast at $32^{\circ} \mathrm{C}$ compared with at $25^{\circ} \mathrm{C}$, so these batches developed to comparable developmental stages. We found that the embryos kept at $32^{\circ} \mathrm{C}$ had more marked cell clusters than the embryos kept at $25^{\circ} \mathrm{C}$ (Fig. 4D, Table S1C). We suggest that, after the heat shock, CRISPR activity is higher or persists for longer at $32^{\circ} \mathrm{C}$ than $25^{\circ} \mathrm{C}$.

\section{Distribution, timing and nature of excisions}

In these experiments we found that we were able to generate marked clones in all regions of the Tribolium embryo, including the anterior head, gnathal, thoracic and abdominal segments. Clones were visible in the ectoderm and the mesoderm of the germband, in developing appendages and in the hindgut (Fig. 5), demonstrating that Valcyrie can be used to generate marked clones in most, if not all, cell populations of the Tribolium embryo.

We were particularly interested to determine whether we could induce flip-outs as early as in late blastoderm or early germband stages, because these are stages when important patterning events, such as establishing segmental boundaries and organ primordia, take place in Tribolium embryos. To address that issue, we subjected late blastoderm embryos $\left(6-7 \mathrm{~h}\right.$ after egg laying, at $\left.32^{\circ} \mathrm{C}\right)$ to a heat shock, and determined the onset of H2B-mCherry expression. We could detect the earliest H2B-mCherry-positive nuclei in early germband stages (after $13 \mathrm{~h}$ at $25^{\circ} \mathrm{C}$ ), showing that Valcyrie constructs can be used to mark clones during these stages. However, we did not detect any H2B-mCherry expression in embryos that were subjected to a 

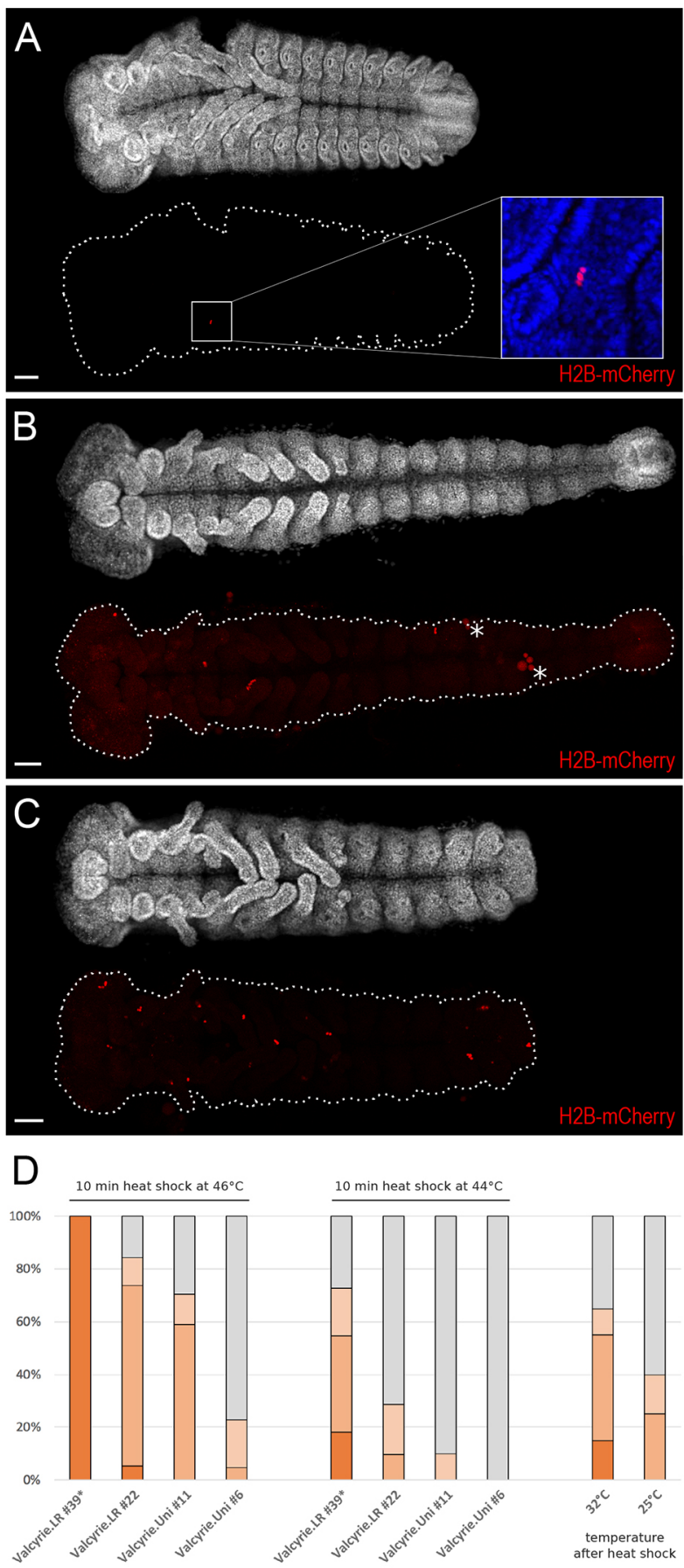

10 min heat shock at $44^{\circ} \mathrm{C}$
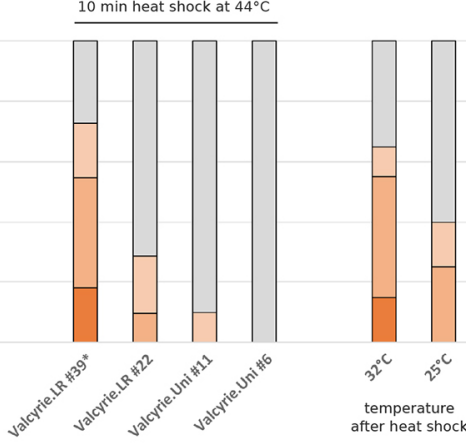

$\square>10$ clones $\quad$ a 2-10 clones $\quad \square 1$ clone $\quad$ nnoclones

after heat shock

heat shock during earlier stages $\left(5-6 \mathrm{~h}\right.$ after egg laying at $32^{\circ} \mathrm{C}$, or younger). The timing of clone induction should correspond to the onset of heat-inducible expression (Schinko et al., 2012).

To molecularly characterise the excision events that take place in the flip-out construct, we extracted genomic DNA from a mix of embryos carrying flip-out clones, used PCR to amplify the region that lies between the paired lox sites, and sequenced several of these DNA fragments. We could detect deletions of variable length spanning that region (Fig. 3C), as is expected for sites that are repaired by non-homologous end joining. In two out of 11 sequenced clones, the deletions extended into the coding sequence of H2B-mCherry. In one other case, part of the $p U b$
Fig. 4. Frequency of cell clones marked by CRISPR-mediated excision. (A-C) Tribolium embryos bearing different numbers of cell clones marked by the Valcyrie approach. The top of each panel shows the morphology of the embryo using Hoechst 34580 staining, marking DNA in all nuclei. The bottom of each panel shows H2B-mCherry fluorescence (red) marking the cell clones. Embryos were categorised as having one cell cluster (A), two to ten cell clusters (B) or more than ten clusters (C). Asterisks indicate autofluorescence in yolk cells. Inset in A shows magnified area with marked clone. (D) The frequency of marked clones is influenced by the sequence of the CRISPR gRNAs, the insertion site of the transgenes, the strength of the heat shock and the incubation temperature after the heat shock. Valcyrie.LR lines yield a higher number of clones per embryo than the Valcyrie.Uni lines, consistent with the degree of complementarity between the gRNAs and the lox targets (see Fig. 3B). The frequency of clones can also be modulated by the heat shock that induces the expression of Cas $9\left(10 \mathrm{~min}\right.$ at $44^{\circ} \mathrm{C}$ versus $\left.46^{\circ} \mathrm{C}\right)$ or by the incubation temperature after the heat shock $\left(25^{\circ} \mathrm{C}\right.$ versus $\left.32^{\circ} \mathrm{C}\right)$. The graphs are based on data shown in Table S1. The frequencies of the Valcyrie.LR \#39 line have been corrected, as half of the embryos do not carry the Valcyrie transgenes. Scale bars: $50 \mu \mathrm{m}$.

promoter was deleted. These results show that, whereas the majority of flip-outs generate marked clones, some Valcyrie excisions can also lead to the loss of both mYFP and H2B-mCherry marker expression.

\section{DISCUSSION}

We have presented a novel method for generating marked cell clones, which relies on CRISPR. Compared with conventional recombinase-mediated clonal marking, our method provides additional means to control the frequency of clonal labelling, by manipulating CRISPR activity; this can be tuned in a predictable fashion by manipulating the degree of sequence complementarity between the CRISPR gRNAs and their targets. The method is highly versatile; the flexible design of gRNAs allows it to be applied on any target construct, including existing constructs with paired lox, $F R T$ or $a t t P / B$ sites, but also on new sites that are introduced using transgenes or on sites found naturally in the genome (see below).

We demonstrated the Valcyrie approach in the beetle $T$. castaneum, an experimental model that is used for comparative studies of development and for studies on pest control. The genetic tools and resources that are available in this species have expanded significantly in recent years, to inducible gene expression, the GAL4/UAS system, a complete genome sequence, large RNAi and enhancer trapping screens, and CRISPR-mediated genome editing (Gilles et al., 2015; Richards et al., 2008; Schinko et al., 2012, 2010; Schmitt-Engel et al., 2015; Trauner et al., 2009). However, clonal marking tools, which would allow us to mark and to track individual cell clones, have been lacking. Our initial effort to establish clonal analysis using a conventional Cre/lox flip-out approach was hampered by the leaky activity of the Cre recombinase, which led to uncontrolled clonal labelling. The Valcyrie approach allows us to overcome this problem, giving us control over the timing and frequency of clonal labelling.

Using Valcyrie, we have shown that the frequency of excision events can be tuned in several ways: by designing gRNAs that have optimal or sub-optimal matches with their target sites (i.e. the Valcyrie.LR or the Valcyrie.Uni constructs), by choosing different transgene insertions, and by modulating the temperature of the heat shock and post-heat-shock treatments. Using the Valcyrie.Uni \#6 and \#11 lines, we have found conditions in which we have reliably generated embryos that carry a single or a few marked clones. Additional levels of control can be envisaged by employing different promoters to drive the expression of Cas9 and/or gRNAs. 

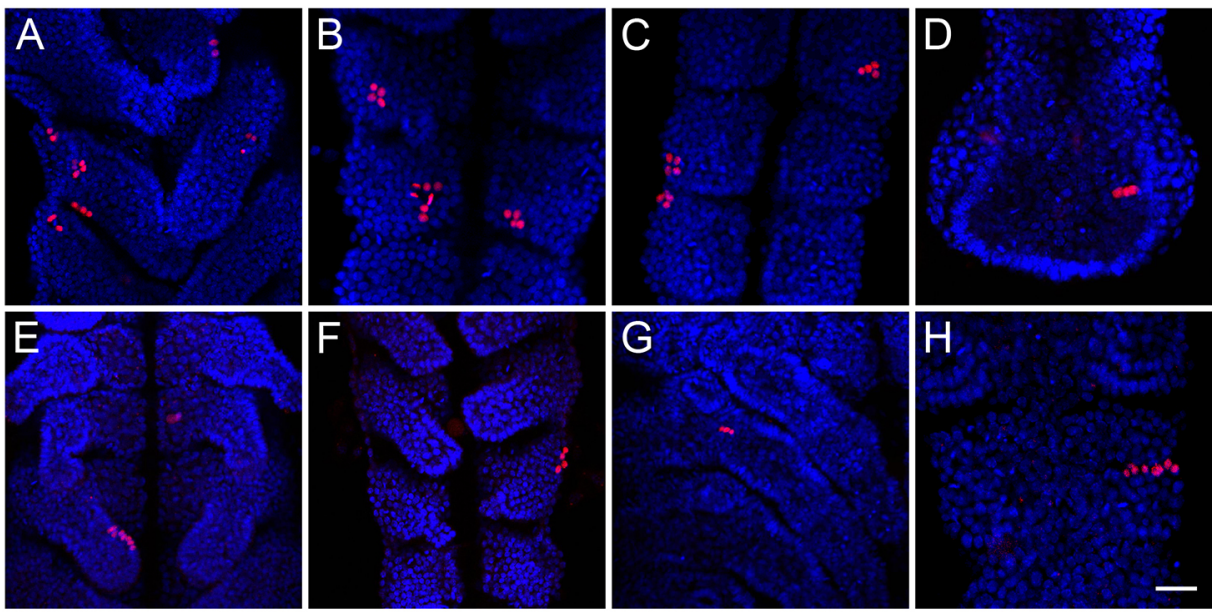

Fig. 5. Cell clones marked by CRISPRmediated excision in Tribolium embryos. (A-H) High magnification views of H2BmCherry-marked cell clones (red) in Tribolium embryos at elongated germband stages. Cell clones marked by CRISPR-mediated excision at late blastoderm stages typically consist of two to eight cells at elongated germband stages. Images include embryos with several marked clones in thoracic and abdominal segments (A-C), and with single marked clones in the posterior mesoderm (D), leg ectoderm (E), abdominal ectoderm $(F)$, leg mesoderm $(G)$ and abdominal mesoderm $(\mathrm{H})$. Note that image in $\mathrm{G}$ is the same as in inset of Fig. 4A. Hoechst 34580 marks DNA in all nuclei (blue). Scale bars: $20 \mu \mathrm{m}$.
We applied Valcyrie to lox flip-out constructs, but the same approach could be used in any experiment that requires excision of a DNA fragment. To perform genetic manipulations within the marked clones, the H2BmCherry marker could be co-expressed with effector transgenes using ribosomal skipping peptides (Szymczak-Workman et al., 2012; the P2A peptide is functional in Tribolium, see Fig. S1). In established model organisms it can be used on existing FRT or lox flip-out constructs, including constructs that are used for mosaic/clonal analysis of gene functions, to finetune the frequency of excision. In principle, CRISPR-mediated excision could also be extended to perform clonal analysis in the absence of flip-out transgenes, by targeting sequences already present in the genome. For example, target sites flanking a cisregulatory element or an exon could be used to excise that element in specific cell populations (using an appropriate driver for Cas9) or in random cell clones. The major challenges in that case will be finding ways to mark the excision events and identifying CRISPR target sites that have no phenotype when individually mutated (e.g. targeting intergenic or intronic regions).

Our method of CRISPR-mediated clonal analysis may be of particular interest in experimental models in which conventional Cre/ lox- and Flp/FRT-based clonal analysis tools are not yet available, but in which transgenesis and CRISPR have been established, such as Nematostella vectensis and Parhyale hawaiensis (Ikmi et al., 2014; Renfer and Technau, 2017; Pavlopoulos and Averof, 2005; Martin et al., 2016). The tunability of Valcyrie provides a straightforward way of adapting and optimising clonal analysis tools in diverse experimental settings and organisms.

\section{MATERIALS AND METHODS}

\section{Cre/lox and Valcyrie constructs}

The Tc-hsp68:Cre construct was generated by cloning the Cre coding sequence under the heat-inducible promoter of the $h s p 68$ gene, including the endoHSE upstream region, the core promoter region (bhsp68) and the 3' UTR of hsp68 (Schinko et al., 2012).

The $p U b:$ lox-mYFP-lox-H2BmCherry construct was generated by cloning the lox-mYFP-lox-H2BmCherry flip-out cassette under the Tribolium $p U b$ element, a $940 \mathrm{bp}$ DNA fragment that includes the promoter, the 5' UTR and the first intron of the Tribolium polyubiquitin gene (see below). Each coding sequence is followed by the SV40 transcriptional termination and polyadenylation signal.

The heat-inducible Cas 9 transgene (Tc-hsp68:Cas 9 ) was generated by cloning the Streptococcus pyogenes Cas9 coding sequence under the heatinducible promoter of the $h s p 68$ gene, including the endoHSE upstream region, the bhsp 68 core promoter region and the 3' UTR of hsp68 (Gilles et al., 2015; Schinko et al., 2012).
To generate the three gRNA transgenes U6b:Lox.Uni, U6b:Lox.R and U6a:Lox.L, we synthesised oligonucleotides that corresponded to each gRNA targeting sequence and cloned these into $\mathrm{p}(\mathrm{U} 6 \mathrm{~b}-\mathrm{BsaI}-\mathrm{gRNA})$ or p(U6a-BsaI-gRNA), as described in Gilles et al., 2015. For Lox.Uni we used oligonucleotides TTCGTTCGTAATAACTTCGTATA and AAACTATACGAAGTTATTACGAA, for Lox.R we used oligonucleotides TTCGATCGCAATAACTTCGTATA and AAACTATACGAAGTTATTGCGAT, and for Lox.L we used oligonucleotides AGTGTACGTAATAACTTCGTATA and AAACTATACGAAGTTATTACGTA.

To generate the Valcyrie.Uni and Valcyrie.LR constructs, we amplified each gRNA transgene using PCR and cloned them into the plasmid carrying the Tc-hsp68:Cas 9 transgene, as illustrated in Fig. 3A.

To generate transgenic lines, these constructs were cloned into the piggyBac transposon vector $p B a c\left[3 x P 3-g T c^{\prime} v\right]$ (Addgene plasmid \#86446, deposited by Gregor Bucher). All constructs were made using a combination of conventional cloning and gene synthesis. Plasmids and annotated sequences are available at Addgene (www.addgene.org).

\section{Transgenic lines}

Stable transgenic lines were generated as described previously (Berghammer et al., 2009) in a Tribolium stock of vermilion ${ }^{\text {white }}$ background (Lorenzen et al., 2002). Transgenic lines carrying single copies of $p B a c[3 x P 3-g T c$ 'v; Tc-hsp68:Cre], pBac [3xP3-gTc'v; pUb:lox$m$ YFP-lox-H2BmCherry], pBac [3xP3-gTc'v; Valcyrie.LR] (transgenic line \#22) and pBac[3xP3-gTc'v; Valcyrie.Uni] (transgenic lines \#6 and \#11) were established and kept as homozygous lines. The insertion of pBac [3xP3-gTc'v; Valcyrie.LR] in Valcyrie.LR transgenic line \#39 is homozygous lethal and was therefore kept in a heterozygous state.

\section{Tribolium promoters}

To identify cis-regulatory elements that are capable of driving uniform, ubiquitous expression of transgenes in early embryos of Tribolium, we cloned and tested the following DNA fragments: (1) a $440 \mathrm{bp}$ fragment of alpha tubulin, cloned by PCR using the primers TGGCCGGCCTGCAGTGAACGGTTATGATGG (including an FseI restriction site) and GGTATACTATACGAAGTTATGGTAGTTGAGTTTTACAAATTAC (including a $20 \mathrm{bp}$ adaptor sequence to facilitate cloning); (2) a $708 \mathrm{bp}$ fragment of RPS3, cloned by PCR using the primers ATGGCCGGCCTCAACGTATGTTGTCAAACC (including an FseI restriction site) and ATGGATCCGACGTTCTAAATGGAAAAGG (including a BamHI restriction site); (3) a 269 bp fragment of RPS18, cloned by PCR using the primers TTGGCCGGCCTGTCAGCGGGACATTGAC (including an FseI restriction site) and TTAGATCTGCAACCAATGCACAAACAAG (including a $B g l \mathrm{II}$ restriction site); (4) a $939 \mathrm{bp}$ fragment of polyubiquitin $(p U b)$, cloned by PCR using the primers TTGGCCGGCCTTTTCTTTGTCCCAAATGACC (including an FseI restriction site) and TAAGATCTGCAACGACACAAAAAATTAC (including a BamHI restriction site). 
These fragments were cloned upstream of the lox-mYFP-lox$H 2 B m C h e r r y$ flip-out cassette and the resulting constructs were introduced in the $p B a c\left[3 x P 3-g T c^{\prime} v\right]$ transgenesis vector (see above). The activities of the RPS3, RPS 18 and $p U b$ constructs were compared by observing mYFP fluorescence in Tribolium embryos $24 \mathrm{~h}$ after injection. $R P S 3$ and RPS18 gave weak fluorescence compared with $p U b$, which gave very strong fluorescence. Stable transgenic lines were then generated for the $p U b$ and alpha tubulin constructs. $p U b$ line $153 \# 13$ gave the strongest uniform expression and was thus chosen for subsequent experiments. We note that all the Tribolium 'ubiquitous' promoters that we have tested (including the previously published EFA fragment; Sarrazin et al., 2012) show patchy expression in late germband stages. As there are no consistent patterns, we ascribe this to noisy zygotic expression.

\section{Generating and screening marked clones}

To generate clones using the Cre/lox approach, we crossed males homozygous for the Tc-hsp 68:Cre transgene (transgenic line CR02) to females homozygous for $p U b$ :lox-mYFP-lox-H2BmCherry (transgenic line 153\#13). We collected embryos from various stages and subjected them to a heat shock for $5 \mathrm{~min}$ at $44^{\circ} \mathrm{C}$. Embryos were then allowed to develop overnight before fixation.

To generate clones using the Valcyrie approach, we crossed males carrying the Valcyrie.LR or Valcyrie.Uni constructs to females homozygous for pUb:lox-mYFP-lox-H2BmCherry (transgenic line 153\#13). We collected embryos from various stages (early germband to late germband elongation) and subjected them to a heat shock for $10 \mathrm{~min}$ at $46^{\circ} \mathrm{C}$. Unless otherwise stated, embryos were allowed to develop for an additional $12 \mathrm{~h}$ at $32^{\circ} \mathrm{C}$ before fixation. We screened the fixed embryos for native H2BmCherry expression using a Zeiss LSM 780 confocal microscope. As a control, we screened 20 embryos from the same crosses that were collected and fixed as described above but were not subjected to a heat shock.

\section{Acknowledgements}

We thank Manon Peleszezak and Benjamin Boumard for their work to characterise the Cre/lox system in Tribolium; Agnès Vallier for technical help; Peter Kitzmann and Gregor Bucher for communicating unpublished data, plasmids and transgenic lines; and Vera Hunnekuhl-Terblanche, Maura Strigini, Jordi Casanova, Luke Hayden and Marco Grillo for helpful comments on the manuscript.

\section{Competing interests}

A.G. and J.S. are co-founders of TriGenes gUG, a not-for-profit platform providing services to the Tribolium community.

\section{Author contributions}

Conceptualization: A.F.G., J.B.S.; Investigation: A.F.G., J.B.S., M.I.S.; Resources: C.E.; Writing - original draft: M.A.; Writing - review \& editing: A.F.G., J.B.S., M.I.S., C.E.; Supervision: A.F.G., J.B.S., M.A.; Project administration: A.F.G., M.A.; Funding acquisition: M.A.

\section{Funding}

This work was supported by a PhD fellowship from the Université Claude Bernard Lyon 1 (to A.F.G.), by a post-doctoral fellowship of the Fondation pour la Recherche Médicale (to J.B.S.) and by a grant from the Agence Nationale de la Recherche (ANR-15-CE13-0014-01).

\section{Supplementary information}

Supplementary information available online at

http://dev.biologists.org/lookup/doi/10.1242/dev.170969.supplemental

\section{References}

Berghammer, A. J., Weber, M., Trauner, J. and Klingler, M. (2009). Red flour beetle (Tribolium) germline transformation and insertional mutagenesis. Cold Spring Harb. Protoc. 2009, pdb.prot5259.

Blanpain, C. and Simons, B. D. (2013). Unravelling stem cell dynamics by lineage tracing. Nat. Rev. Mol. Cell Biol. 14, 489-502.

Brook, W. J., Diaz-Benjumea, F. J. and Cohen, S. M. (1996). Organizing spatial pattern in limb development. Annu. Rev. Cell Dev. Biol. 12, 161-180.

Chen, C.-H., Puliafito, A., Cox, B. D., Primo, L., Fang, Y., Di Talia, S. and Poss, K. D. (2016). Multicolor cell barcoding technology for long-term surveillance of epithelial regeneration in zebrafish. Dev. Cell 36, 668-680.
Cong, L., Ran, F. A., Cox, D., Lin, S., Barretto, R., Habib, N., Hsu, P. D., Wu, X., Jiang, W., Marraffini, L. A. et al. (2013). Multiplex genome engineering using CRISPR/Cas systems. Science 339, 819-823.

Frickenhaus, M., Wagner, M., Mallik, M., Catinozzi, M. and Storkebaum, E. (2015). Highly efficient cell-type-specific gene inactivation reveals a key function for the Drosophila FUS homolog cabeza in neurons. Sci. Rep. 5, 9107.

Fu, Y., Foden, J. A., Khayter, C., Maeder, M. L., Reyon, D., Joung, J. K. and Sander, J. D. (2013). High-frequency off-target mutagenesis induced by CrlsPrCas nucleases in human cells. Nat. Biotechnol. 31, 822-826.

Garcia-Bellido, A., Ripoll, P. and Morata, G. (1973). Developmental compartmentalisation of the wing disk of Drosophila. Nat. New Biol. 245, 251-253.

Gilles, A. F. and Averof, M. (2014). Functional genetics for all: engineered nucleases, CRISPR and the gene editing revolution. EvoDevo 5, 1-13.

Gilles, A. F., Schinko, J. B. and Averof, M. (2015). Efficient CRISPR-mediated gene targeting and transgene replacement in the beetle Tribolium castaneum. Development 142, 2832-2839.

Golic, K. G. and Lindquist, S. (1989). The FLP recombinase of yeast catalyzes site-specific recombination in the Drosophila genome. Cell 59, 499-509.

Griffin, R., Binari, R. and Perrimon, N. (2014). Genetic odyssey to generate marked clones in Drosophila mosaics. Proc. Natl. Acad. Sci. USA 111, 4756-4763.

Hotta, Y. and Benzer, S. (1972). Mapping of behaviour in Drosophila mosaics. Nature 240, 527-535.

Hsu, P. D., Scott, D. A., Weinstein, J. A., Ran, F. A., Konermann, S., Agarwala, V., Li, Y., Fine, E. J., Wu, X., Shalem, O. et al. (2013). DNA targeting specificity of RNA-guided Cas9 nucleases. Nat. Biotechnol. 31, 827-832.

Hwang, W. Y., Fu, Y., Reyon, D., Maeder, M. L., Tsai, S. Q., Sander, J. D., Peterson, R. T., Yeh, J.-R. J. and Joung, J. K. (2013). Efficient genome editing in zebrafish using a CRISPR-Cas system. Nat. Biotechnol. 31, 227-229.

Ikmi, A., McKinney, S. A., Delventhal, K. M. and Gibson, M. C. (2014). TALEN and CRISPR/Cas9-mediated genome editing in the early-branching metazoan Nematostella vectensis. Nat. Commun. 5, 5486.

Janning, W. (1978). Gynandromorph fate maps in Drosophila. Results Probl. Cell Differ. 9, 1-28.

Jiang, W., Bikard, D., Cox, D., Zhang, F. and Marraffini, L. A. (2013). RNA-guided editing of bacterial genomes using CRISPR-Cas systems. Nat. Biotechnol. 31 233-239.

Jinek, M., Chylinski, K., Fonfara, I., Hauer, M., Doudna, J. A. and Charpentier, E. (2012). A programmable dual-RNA-guided DNA endonuclease in adaptive bacterial immunity. Science 337, 816-821.

Kretzschmar, K. and Watt, F. M. (2012). Lineage tracing. Cell 148, 33-45.

Kuchen, E. E., Fox, S., Barbier de Reuille, P., Kennaway, R., Bensmihen, S., Avondo, J., Calder, G. M., Southam, P., Robinson, S., Bangham, A. et al. (2012). Generation of leaf shape through early patterns of growth and tissue polarity. Science $335,1092-1096$

Lee, T. and Luo, L. (1999). Mosaic analysis with a repressible cell marker for studies of gene function in neuronal morphogenesis. Neuron 22, 451-461.

Livet, J., Weissman, T. A., Kang, H., Draft, R. W., Lu, J., Bennis, R. A., Sanes, J. R. and Lichtman, J. W. (2007). Transgenic strategies for combinatorial expression of fluorescent proteins in the nervous system. Nature 450, 56-62.

Lorenzen, M. D., Brown, S. J., Denell, R. E. and Beeman, R. W. (2002). Cloning and characterization of the Tribolium castaneum eye-color genes encoding tryptophan oxygenase and kynurenine 3-monooxygenase. Genetics 160 225-234

Mali, P., Yang, L., Esvelt, K. M., Aach, J., Guell, M., DiCarlo, J. E., Norville, J. E. and Church, G. M. (2013). RNA-guided human genome engineering via cas9. Science 339, 823-826.

Martin, A., Serano, J. M., Jarvis, E., Bruce, H. S., Wang, J., Ray, S., Barker, C. A. O'Connell, L. C. and Patel, N. H. (2016). CRISPR/Cas9 mutagenesis reveals versatile roles of hox genes in crustacean limb specification and evolution. Curr. Biol. 26, 14-26.

Meyer, S. N., Amoyel, M., Bergantiños, C., de la Cova, C., Schertel, C., Basler, K. and Johnston, L. A. (2014). An ancient defense system eliminates unfit cells from developing tissues during cell competition. Science 346, 1258236-1258236.

Morante, J. and Desplan, C. (2008). The color-vision circuit in the medulla of Drosophila. Curr. Biol. 18, 553-565.

Nicolas, J. F., Mathis, L., Bonnerot, C. and Saurin, W. (1996). Evidence in the mouse for self-renewing stem cells in the formation of a segmented longitudinal structure, the myotome. Development 122, 2933-2946.

Orban, P. C., Chui, D. and Marth, J. D. (1992). Tissue- and site-specific DNA recombination in transgenic mice. Proc. Natl. Acad. Sci. USA 89, 6861-6865.

Pavlopoulos, A. and Averof, M. (2005). Establishing genetic transformation for comparative developmental studies in the crustacean Parhyale hawaiensis. Proc. Natl. Acad. Sci. USA 102, 7888-7893.

Rajewsky, K., Gu, H., Kühn, R., Betz, U. A., Müller, W., Roes, J. and Schwenk, F. (1996). Conditional gene targeting. J. Clin. Investig. 98, 600-603.

Renfer, E. and Technau, U. (2017). Meganuclease-assisted generation of stable transgenics in the sea anemone Nematostella vectensis. Nat. Protoc. 12 1844-1854. 
Richards, S., Gibbs, R. A., Weinstock, G. M., Brown, S. J., Denell, R., Beeman, R. W., Gibbs, R., Bucher, G., Friedrich, M., Grimmelikhuijzen, C. J. et al. (2008). The genome of the model beetle and pest Tribolium castaneum. Nature 452, 949-955.

Richier, B. and Salecker, I. (2014). Versatile genetic paintbrushes: brainbow technologies. WIREs Dev. Biol. 4, 161-180.

Sarrazin, A. F., Peel, A. D. and Averof, M. (2012). A segmentation clock with twosegment periodicity in insects. Science 336, 338-341.

Schinko, J. B., Weber, M., Viktorinova, I., Kiupakis, A., Averof, M., Klingler, M., Wimmer, E. A. and Bucher, G. (2010). Functionality of the GAL4/UAS system in Tribolium requires the use of endogenous core promoters. BMC Dev. Biol. 10, 53.

Schinko, J. B., Hillebrand, K. and Bucher, G. (2012). Heat shock-mediated misexpression of genes in the beetle Tribolium castaneum. Dev. Genes Evol. 222 287-298.

Schmitt-Engel, C., Schultheis, D., Schwirz, J., Ströhlein, N., Troelenberg, N., Majumdar, U., Dao, V. A., Grossmann, D., Richter, T., Tech, M. et al. (2015) The iBeetle large-scale RNAi screen reveals gene functions for insect development and physiology. Nat. Commun. 6, 795.

Shaner, N. C., Campbell, R. E., Steinbach, P. A., Giepmans, B. N. G., Palmer, A. E. and Tsien, R. Y. (2004). Improved monomeric red, orange and yellow fluorescent proteins derived from Discosoma sp. red fluorescent protein. Nat. Biotechnol. 22, 1567-1572.

Snippert, H. J., van der Flier, L. G., Sato, T., van Es, J. H., van den Born, M., Kroon-Veenboer, C., Barker, N., Klein, A. M., van Rheenen, J., Simons, B. D. et al. (2010). Intestinal crypt homeostasis results from neutral competition between symmetrically dividing Lgr5 stem cells. Cell 143, 134-144.

Stern, C. (1936). Somatic crossing over and segregation in Drosophila melanogaster. Genetics 21, 625-730.

Szymczak-Workman, A. L., Vignali, K. M. and Vignali, D. A. A. (2012). Design and construction of 2A peptide-linked multicistronic vectors. CSH Protocols 2012 http://doi.org/10.1101/pdb.ip067876.

Trauner, J., Schinko, J., Lorenzen, M. D., Shippy, T. D., Wimmer, E. A., Beeman, R. W., Klingler, M., Bucher, G. and Brown, S. J. (2009). Large-scale insertional mutagenesis of a coleopteran stored grain pest, the red flour beetle Tribolium castaneum, identifies embryonic lethal mutations and enhancer traps. BMC Biol. $7,73$.

Vincent, C. A., Carpenter, R. and Coen, E. S. (1995). Cell lineage patterns and homeotic gene activity during Antirrhinum flower development. Curr. Biol. 5, 1449-1458. 
Figure S1. Functionality of the viral P2A peptide in Tribolium castaneum

Bicistronic expression of membrane-localized YFP (in green) and Histone2B-mCherry, mediated by the viral P2A peptide in blastoderm and early germband stage embryos of Tribolium castaneum. The two proteins are expressed from the same open reading frame, separated by the PTV1 peptide (Szymczak-Workman et al., 2012). The transgene was stably integrated in the genome. The distinct localization of membrane-YFP and Histone2BmCherry proteins in the plasma membrane and chromatin, respectively, indicates that the P2A peptide is functional (mediates ribosomal skipping) in Tribolium.
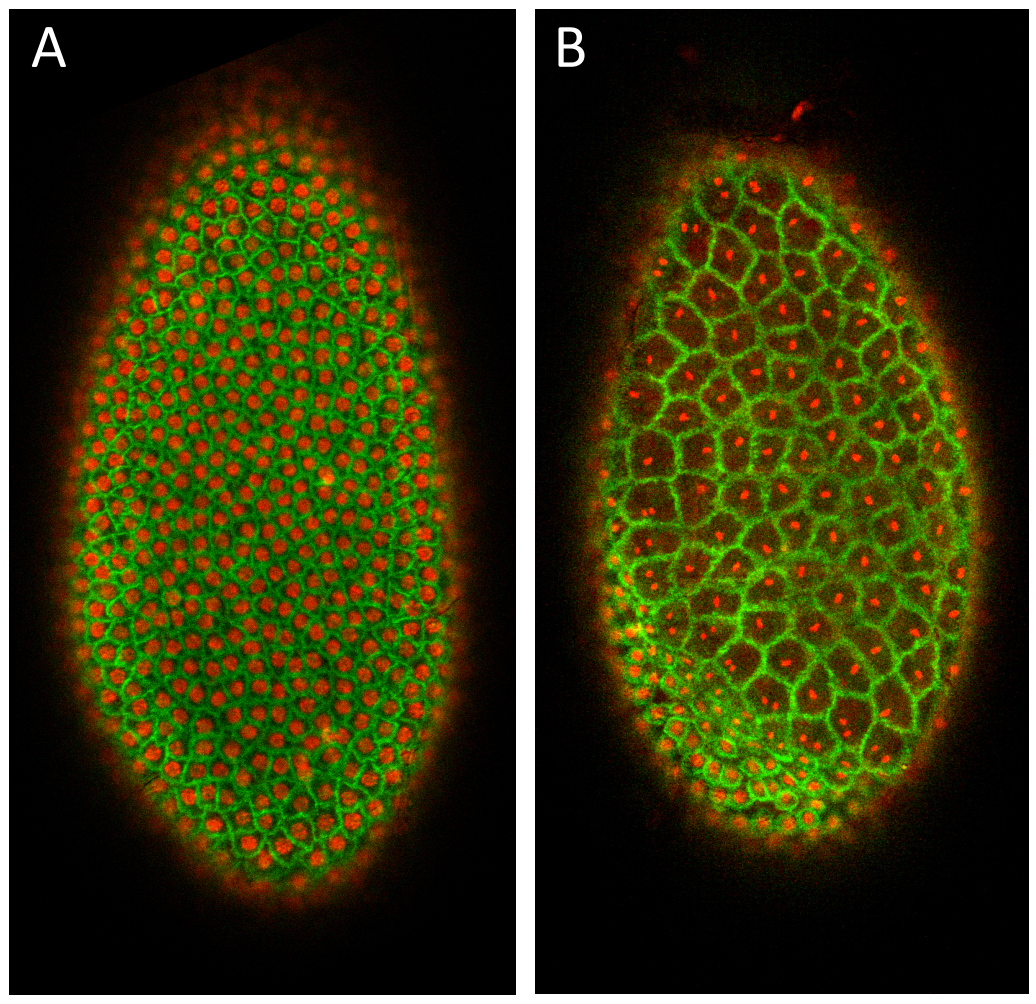
Table S1. Frequency of marked cell clones. The number of distinct clusters of H2BmCherry-expressing cells was scored per embryo. We classified embryos in four categories: embryos with more than 10 clusters per embryo, 2-10 clusters, one cluster, or none. Each cluster was taken to represent a cell clone.

$$
>10 \text { clones 2-10 clones } 1 \text { clone no clones }
$$

A. Embryos with marked clones after 10 min heat shock at $46^{\circ} \mathrm{C}$

\begin{tabular}{llrrrr}
\hline Valcyrie.LR \#22 & $(\mathrm{n}=19)$ & $5 \%$ & $68 \%$ & $11 \%$ & $16 \%$ \\
Valcyrie.LR \#39* & $(\mathrm{n}=15)$ & $60 \%$ & $0 \%$ & $0 \%$ & $40 \%$ \\
Valcyrie.Uni \#6 & $(\mathrm{n}=22)$ & $0 \%$ & $5 \%$ & $18 \%$ & $77 \%$ \\
Valcyrie.Uni \#11 & $(\mathrm{n}=17)$ & $0 \%$ & $59 \%$ & $12 \%$ & $29 \%$
\end{tabular}

B. Embryos with marked clones after 10 min heat shock at $44^{\circ} \mathrm{C}$

\begin{tabular}{lllccc}
\hline Valcyrie.LR \#22 & $(\mathrm{n}=21)$ & $0 \%$ & $10 \%$ & $19 \%$ & $71 \%$ \\
Valcyrie.LR \#39* & $(\mathrm{n}=11)$ & $9 \%$ & $18 \%$ & $9 \%$ & $64 \%$ \\
Valcyrie.Uni \#6 & $(\mathrm{n}=19)$ & $0 \%$ & $0 \%$ & $0 \%$ & $100 \%$ \\
Valcyrie.Uni \#11 & $(\mathrm{n}=20)$ & $0 \%$ & $0 \%$ & $10 \%$ & $90 \%$ \\
\hline C. Effect of post-heat-shock temperature (after 10 min heat shock & \multicolumn{2}{c}{$46^{\circ} \mathrm{C}$, Valcyrie.LR \#22) } \\
\hline $32^{\circ} \mathrm{C}$ & $(\mathrm{n}=20)$ & $15 \%$ & $40 \%$ & $10 \%$ & $35 \%$ \\
$25^{\circ} \mathrm{C}$ & $(\mathrm{n}=20)$ & $0 \%$ & $25 \%$ & $15 \%$ & $60 \%$ \\
\hline
\end{tabular}

* The Valcyrie.LR \#39 line is heterozygous, therefore approximately $50 \%$ of the embryos in these experiments did not carry the Valcyrie.LR construct. 